\title{
PERFORMANCE EVALUATION OF ETHERNET TRANSMISSION USING M-ARY PULSE AMPLITUDE MODULATION TECHNIQUES
}

\author{
APENA WALIU OLALEKAN ${ }^{1}$, OLASUNKANMI OMOWUMI GRACE ${ }^{2 *}$, \\ SALAKO ANUOLUWAPO ${ }^{3}$ \\ ${ }^{I}$ Department of Computer Engineering, The Federal University of Technology, Akure, \\ Nigeria \\ ${ }^{2}$ Department of Electrical/Electronic Engineering, Olabisi Onabanjo University, Ago-Iwoye, \\ Nigeria \\ ${ }^{3}$ Department of Electrical/Electronic Engineering, The Federal University of Technology, \\ Akure, Nigeria
}

\begin{abstract}
Low installation costs and high data rates reaching up to $10 \mathrm{Gbps}$, characterized Ethernet as the local area network (LAN) technology of choice to satisfy the increasing need for high-speed data transmission in packet-based networks. As demand for high speeds in data has increased, copper Ethernet has been integrated to handle these higher speeds. The IEEE 802.3ae* 2002 (10 Gigabit Ethernet) standard is based on data transmission over optical fibre only and in full-duplex mode. This study considered performance evaluation of Pulse Amplitude Modulation (PAM) and multilevel (PAM-16) technology in comparison with other PAM versions was carried out to investigate copper Ethernet with respect to higher speed characteristic and error performance. The evaluation was carried out in MATLAB R2017b simulation environment; this provides calculated bit error rates (BER) of the considered modulation schemes under different channel conditions. The results show that PAM-16 has a BER of $10^{-12}$.which is significantly lower than that of PAM-2 and PAM-4. Additionally, Hamming code were used to detect and correct errors that are inherent in the design and the efficiency of each level of PAM used was analyzed.
\end{abstract}

Keywords: Amplitude, Error, Ethernet, MATLAB R2017b, Modulation.

\section{INTRODUCTION}

Pulse amplitude modulation (PAM) is a form pulse modulation, where the signal is sampled at regular interval with respect to the amplitude of the modulating signal. M-ary pulse modulation technique exists in multilevel in which data is transmitted by varying the amplitude of the pulses used to represent the message signal. In the case of analogue pulse amplitude modulation signals, the number of pulse amplitudes can be infinite. Pulse amplitude modulation finds application in digital data transmissions that are broadband to accommodate high data rate. Pulse amplitude modulation is used in the popular Ethernet communication standard [5].

Modern communication and entertainment adopted Ethernet cables over other conventional connection to connect devices that are wired in local area networks for smooth connectivity. This give the reason for copper Ethernet cables like1000BASE-T and 10GBASE-T to takeaccount of crosstalk, return loss impairments, together with near and far-end crosstalk having multiple disturbers in the cabling.

As a result of greater bandwidth of 10GBASE-T signals, significantly, more crosstalk exists from adjacent cables than 1000BASE-T (known as alien crosstalk which is abbreviated as AXT). Noise and interference such as background noise and alien near-end crosstalk (NEXT) interference from other cables can also reduce the received

*Corresponding author, email: grace.olasunkanmi@oouagoiwoye.edu.ng

(C) 2020 Alma Mater Publishing House 
Signal-Noise Ratio (SNR). To meet the desired throughput (e.g., 10Gbps) and target BER requirements (e.g., 10 ${ }^{12}$ ), a DSP transceiver has to perform a significant amount of digital processing operations, which include channel equalization, channel coding, and noise cancellation such as echo and crosstalk interferences including NEXT and/or FEXT.

10GBASE-T uses 16-level PAM (Pulse Amplitude Modulation) signalling in each pair. This uses 4-pair transmission and was designed to support up to 100 metres of Category 6A or higher cabling. The aggregate data rate of $10 \mathrm{Gbit} / \mathrm{s}$ is achieved by transmitting $2.5 \mathrm{Gbit} / \mathrm{s}$ over each cable pair. 16 levels of Pulse Amplitude Modulation are derived from 4 binary bits $\left(\log _{2} 16\right)$, and each 16-level PAM value is referred to as a PAM16 symbol. A two-dimensional (2D) code is created using a pair of adjacent PAM16 symbols [2].

1000BASE-T project specifically requires operation on four pair, 100-Ohm Category 5 balanced copper cabling as defined by ANSI/TIA/EIA-568-A, or its equivalent as built from material specified by ISO/IEC 11801:1995 that meets the channel performance parameters specified in ANSI/TIA/EIA-568-A ANNEX E.

Transmitting a $1000 \mathrm{Mb} / \mathrm{s}$ data stream over four pairs of Category 5twisted pair cables could present several design challenges to the transmission system designer (and standards developer). The challenges are due to signal attenuation and distortions such as echo, return loss, and crosstalk characteristics of cables, as well as electromagnetic emissions and susceptibility as shown in Figure 1.

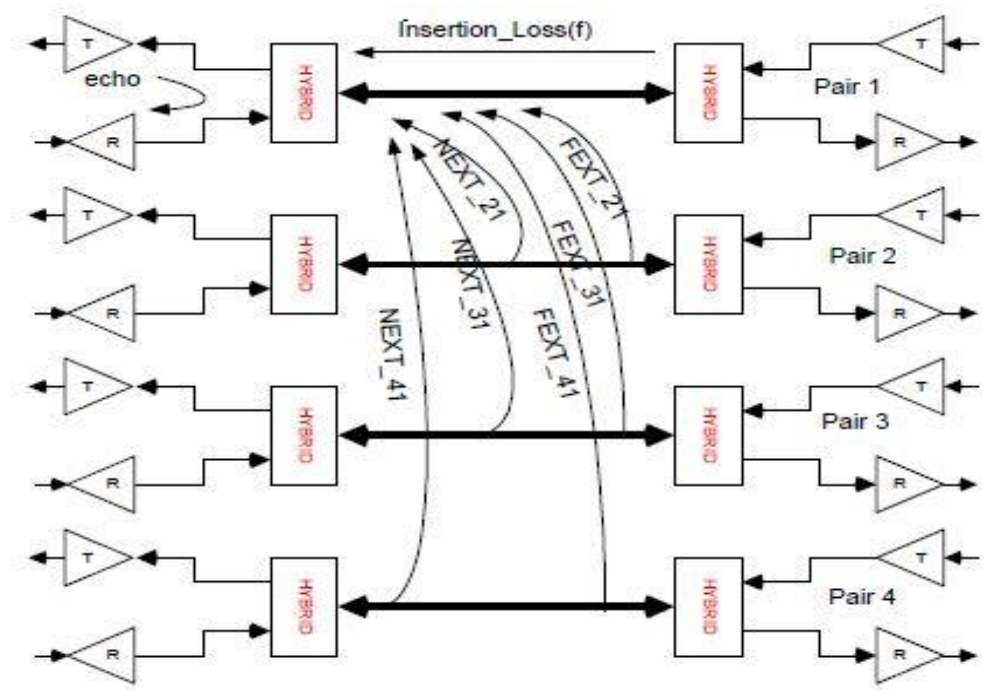

Fig. 1. CAT 5 Impairment

10GBASE-T is 10 times faster and more sophisticated which cancels SelfCross talk and Echo than 1000BASE-T [6].

For data-rates of $10 \mathrm{~Gb} / \mathrm{s}$, the signal needs to be transmitted at frequencies up to a minimum of $500 \mathrm{MHz}$, as compared with Category 6 transmissions which run up to $200 \mathrm{MHz}$. To achieve a promise communication link at these high frequencies over UTP copper requires significant changes in the way the channel components are designed. At these high frequencies, all components of a10G channel begin to emit electromagnetic fields, which negatively impact the channels that are closest in physical proximity. This interaction between one channel and its neighboring channels is called Alien Near End Cross Talk (ANEXT). Overcoming this gap posed a challenge of Augmented Cat 6 and Cat 7 standard [4]. 


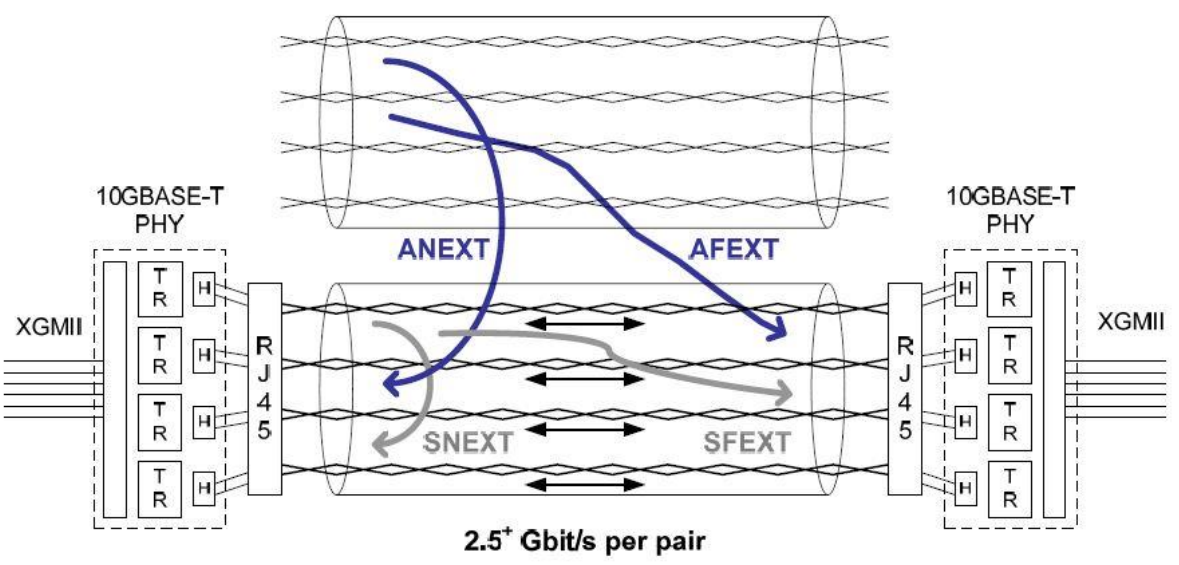

Fig. 2. 10Gbase-T category 6 cable

For data-rates of $10 \mathrm{~Gb} / \mathrm{s}$ (Figure 2), the signal needs to be transmitted at frequencies up to a minimum of 500 $\mathrm{MHz}$, as compared with Category 6 transmissions which run up to $200 \mathrm{MHz}$. To achieve a promise communication link at these high frequencies over UTP copper requires significant changes in the way the channel components are designed. At these high frequencies, all components of a10G channel begin to emit electromagnetic fields, which negatively impact the channels that are closest in physical proximity. This interaction between one channel and its neighboring channels is called Alien Near End Cross Talk (ANEXT). Overcoming this gap posed a challenge of Augmented Cat 6 and Cat 7 standard [4].

\section{METHODOLOGY}

The study investigated comparison between PAM-2, PAM-4 and PAM-16 in MATLAB R2017b environment. The comparison between the characteristic's properties were revealed in constellation diagram, gray code mapping, signal-to-noise ratio, hamming code and with respect to BER. All this was developed in MATLAB R2017b simulation and was justified using MATLAB code. PAM-2, PAM-4 and PAM-16 signal performances simulations were compared in MATLAB R2017b, the study present a need to explain the methods of generating a PAM signal in MATLAB. There are several ways of generating PAM signal but this project deplored system object communication tools using PAM modulator for M-ary PAM employed in the study; the procedures (flowcharts) are presented accordingly.

\subsection{Generating PAM-2, PAM-4 and PAM-16 Signal using MATLAB}

To generatee PAM-2, PAM-4 and PAM-16 signal, the flow chart Figure 3 was used to generate a random digital signal data bit.

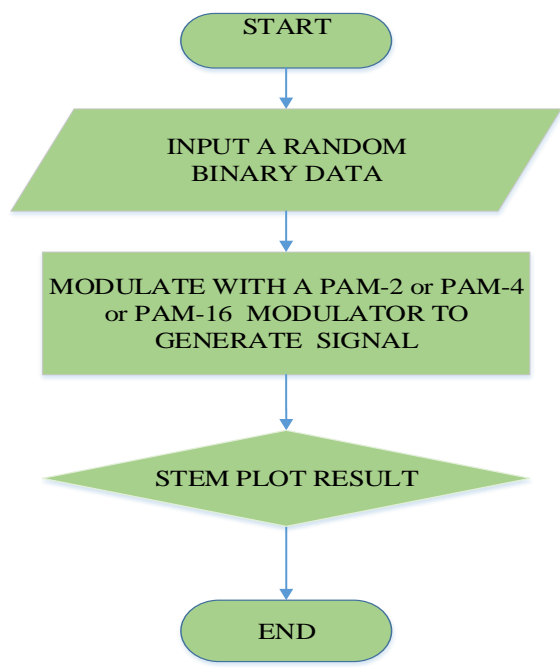

Fig. 3. Flowchart for generation of PAM signal. 


\subsection{Constellation diagram comparison}

There are several approaches to evaluate modulated signal of PAM-2, PAM-4 and PAM-16, the study adopted the use of constellation diagram. The constellation diagram displays output signals as a two-dimensional xy-plane scatter diagram in the complex plane as revealed by the flow chart Figure 4.

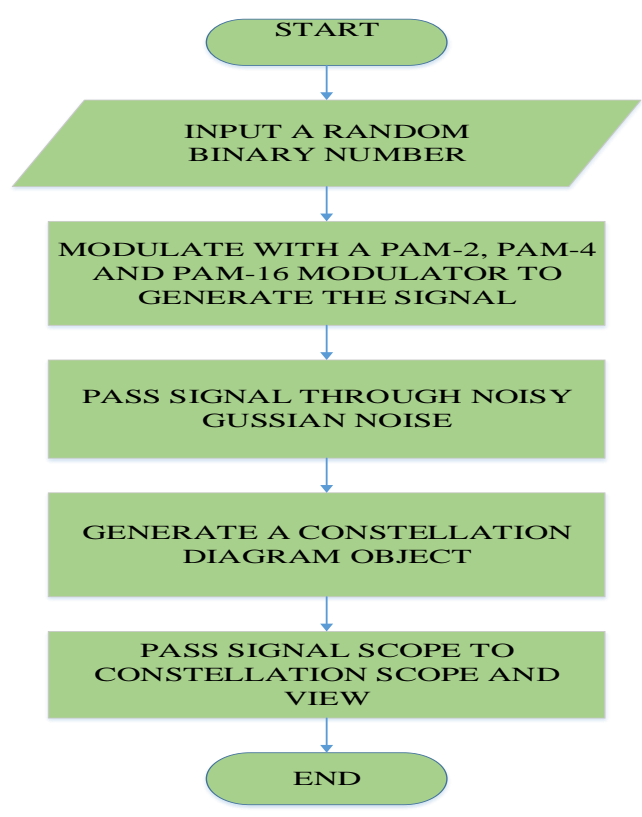

Fig. 4. Flowchart for Constellation diagram

\subsection{Probability of error in PAM-2, PAM-4 and PAM-16 signal}

The study error coding system to support better outcome result by developing mathematical models using equations as illustrated below.

Considering binary PAM baseband signals, with two signal waveforms taken into consideration represented as:

$$
\begin{aligned}
& \mathrm{s} 1(\mathrm{t})=\mathrm{gT}(\mathrm{t}) \\
& \mathrm{s} 2(\mathrm{t})=-\mathrm{gT}(\mathrm{t})
\end{aligned}
$$

where $\mathrm{gT}(\mathrm{t})$ is an arbitrary pulse which is nonzero in the interval $0 \leq \mathrm{t} \leq \mathrm{Tb}$ and zero elsewhere.

Since $\mathrm{s} 1(\mathrm{t})=-\mathrm{s} 2(\mathrm{t})$, these signals are said to be antipodal. The energy in the pulse $\mathrm{gT}(\mathrm{t})$ and the energy per bit $(\mathrm{Eb})$ are equation [1].

In assumption, the two signals have equal likelihood and that signal s1(t) was transmitted. Then, the received signal from the (matched filter or correlation-type) demodulator as presented as equation:

$$
\mathrm{r}=\mathrm{s} 1+\mathrm{n}=\mathrm{Eb}+\mathrm{n}
$$

where, $\mathrm{n}$ represents the Additive White Gaussian noise component which has zero mean and variance.

$$
\sigma^{2}=\mathrm{No} / 2
$$

where, $N_{0=N O I S E}$

If $r>0$, the decision is made in favor of $\mathrm{s} 1(\mathrm{t})$ and if $\mathrm{r}<0$, the decision is made in favor of $\mathrm{s} 2(\mathrm{t})$.

$$
\mathrm{P}(\mathrm{e} \mid \mathrm{s} 1)=\int_{\infty}^{0} \mathrm{p}(\mathrm{r} \mid \mathrm{s} 1) \mathrm{dr}=\frac{1}{\sqrt{\pi \mathrm{N} 0}} \int_{-\infty}^{0} \mathrm{e}^{-\left(\mathrm{r}-\sqrt{\varepsilon_{\mathrm{b}}}\right)^{2}} \mathrm{dr}=\frac{1}{\sqrt{2 \pi}} \int_{-\infty}^{-\sqrt{2 \varepsilon_{\mathrm{b}} / \mathrm{N}_{0}}} \mathrm{e}^{-\mathrm{x}^{2} / 2} \mathrm{dx}=\mathrm{Q}\left(\sqrt{\frac{2 \varepsilon_{\mathrm{b}}}{\mathrm{N}_{0}}}\right)
$$


Similarly, if we assume that $\mathrm{s} 2(\mathrm{t})$ was transmitted, $\mathrm{r}=-\sqrt{\mathrm{Eb}}+\mathrm{n}$.

Since the signals $\mathrm{s} 1(\mathrm{t})$ and $\mathrm{s} 2(\mathrm{t})$ are equally likely to be transmitted, the average probability of error is:

$$
\mathrm{P}_{\mathrm{b}}=\frac{1}{2} \mathrm{p}\left(\mathrm{e} / \mathrm{s}_{1}\right)+\frac{1}{2} \mathrm{p}\left(\mathrm{e} / \mathrm{s}_{2}\right) \Rightarrow p_{b=Q}\left(\sqrt{\frac{2 E_{b}}{N_{0}}}\right)
$$

In general, for a PAM-L signal, the probability of error is given below:

$$
\mathrm{Pe}=\frac{\mathrm{L}-1}{\mathrm{~L}} \operatorname{erfc}\left(\frac{\mathrm{Q}}{\sqrt{2(\mathrm{~L}-1)}}\right)
$$

where $\mathrm{L}$ is the number of levels as shown in the above equation.

If $\mathrm{L}$ is 16 such as in PAM-16 then:

$$
\mathrm{Pe}=\frac{15}{16} \operatorname{erfc}(\mathrm{Q} / 15 \sqrt{4})
$$

where $\mathrm{Q}$ is the quality factor [3].

Figure 5 present the Signal-to-noise ratio flow PAM-2, PAM-4 and PAM-16 signal

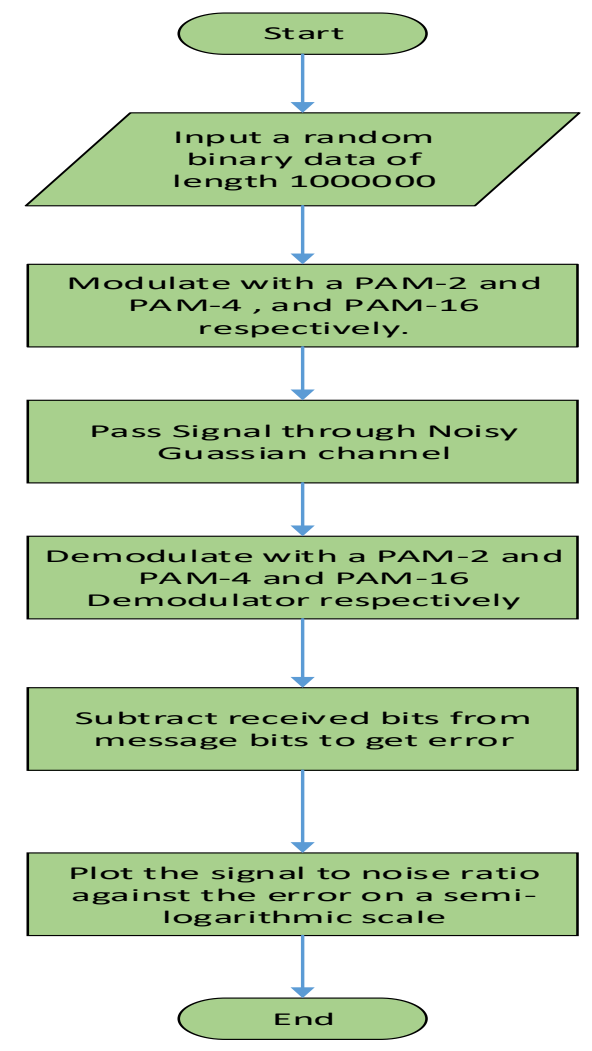

Fig. 5. Signal-to-noise ratio flow chart.

\subsection{Hamming Code Algorithm}

The Hamming detecting and correcting code are represented in Figure 6. 


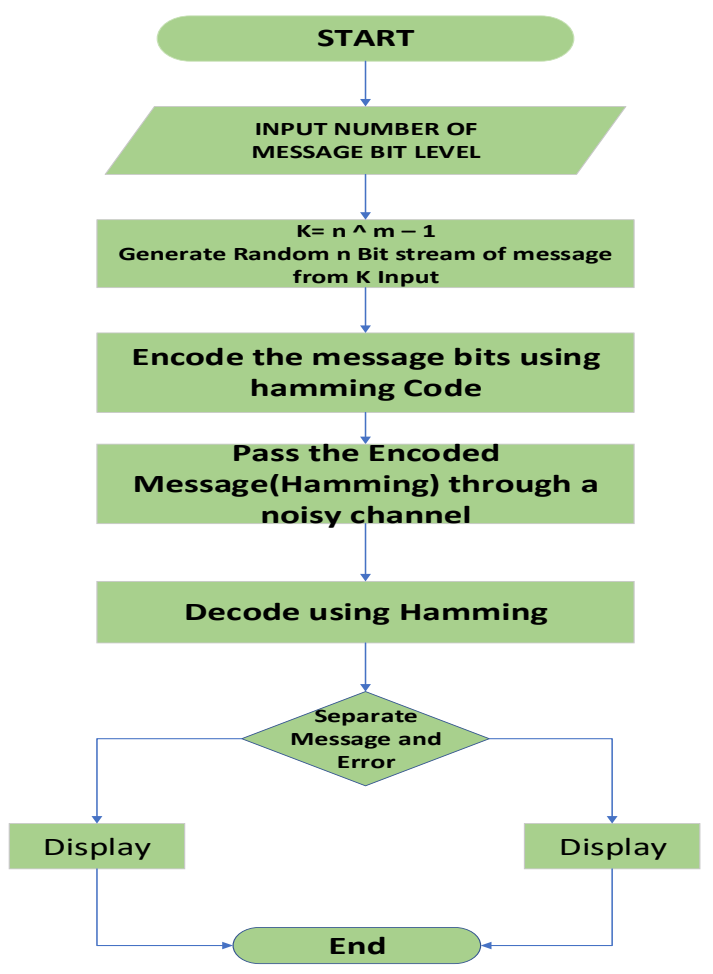

Fig. 6. Flowchart for Hamming detecting and correcting code.

\section{RESULTS AND DISCUSSION}

The characteristics of modulated signal for PAM-2, PAM-4 and PAM-16 are shown; and the graphical result explained each of these signals as shown in Figure 7, 8 and 9 below. PAM was considered as the major modulation technique in the work and supported with other multilevel $(M=2, M=4, M=16)$ modulation respectively. The various multilevel of PAM discussed are presented by transmitting data through varying amplitude of the electrical or electromagnetic pulses for both sampled and quantized signal as shown.

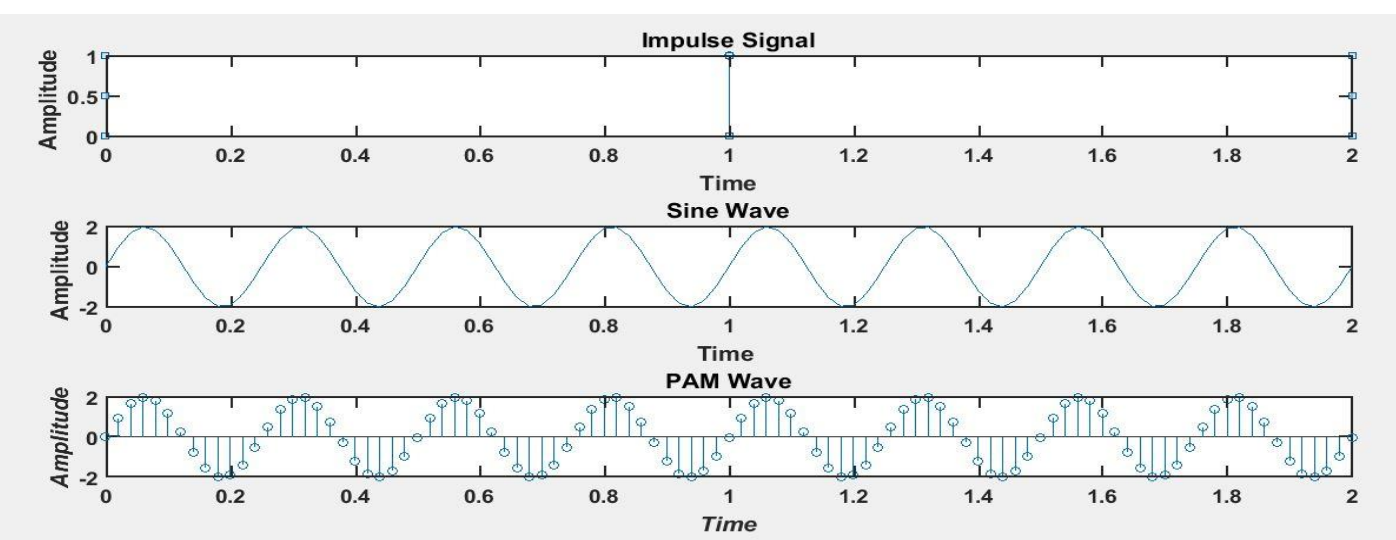

Fig. 7. PAM-2 sampling and Quantizing process. 

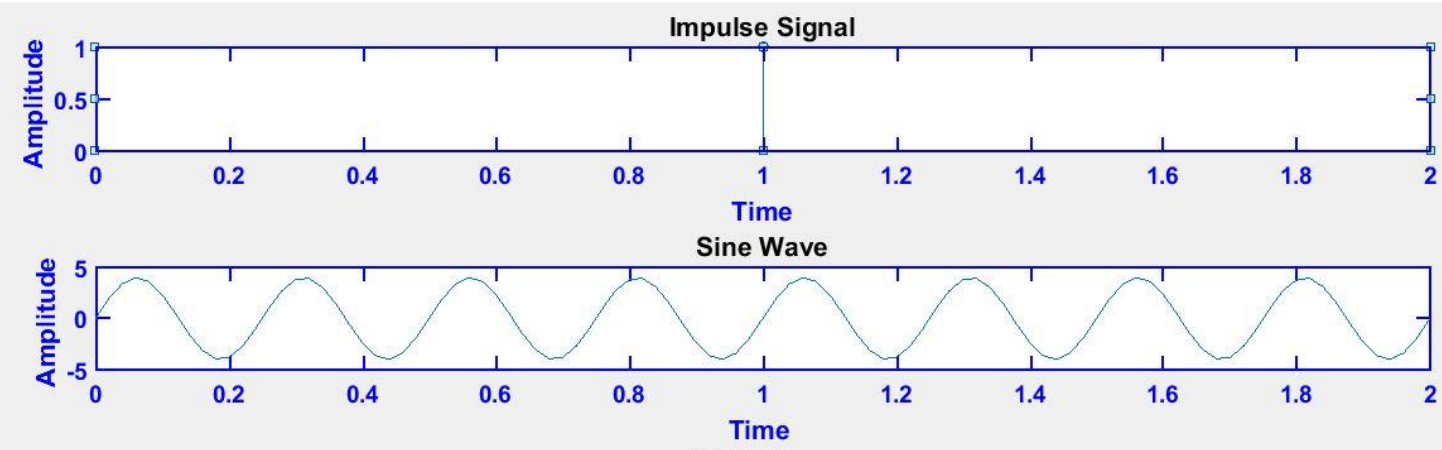

PAM Wave

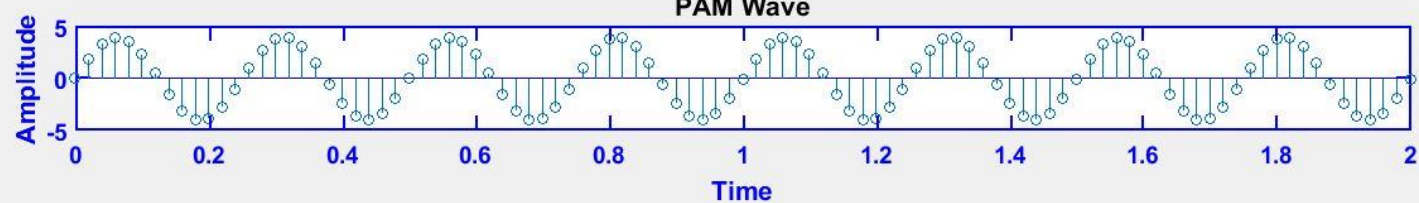

Fig. 8. PAM-4 Sampling and Quantizing process.
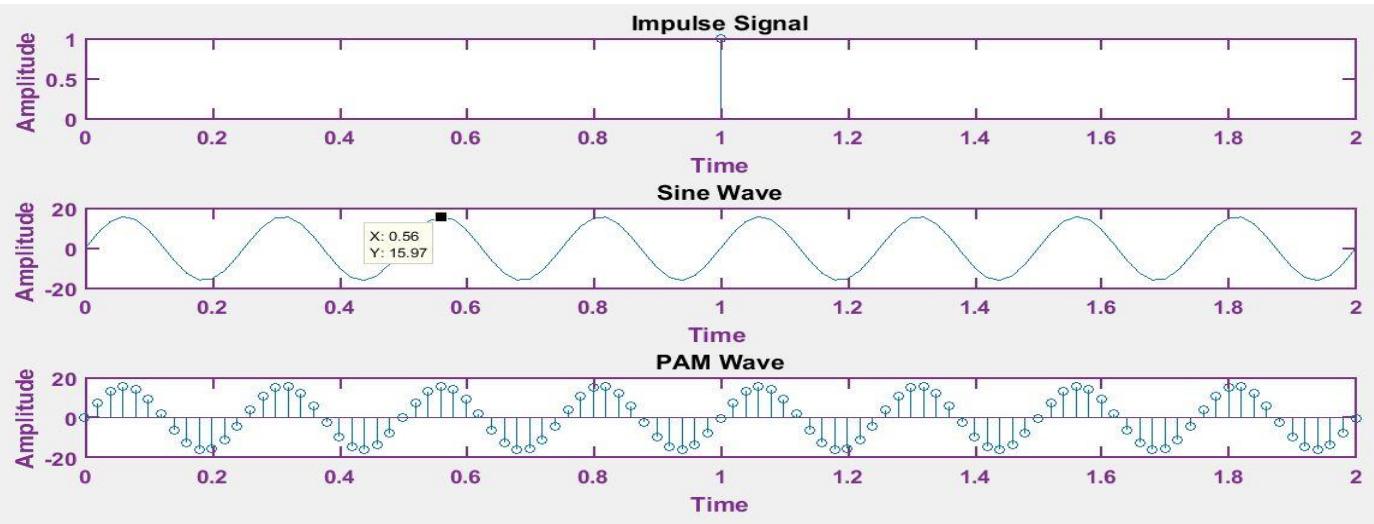

Fig. 9.PAM-16 Sampling and Quantizing process.

\subsection{Constellation Diagram comparison}

The constellation diagram depicts the PAM-2 signal (Figure 10), PAM-4 (Figure 11) and PAM-16 signal (Figure 12) without and with noise respectively. The diagram shows the corresponding distance between the levels, and it is graphically clear that the 2 levels of PAM- 2 are widely spaced compared to adjacent levels in PAM-4 and PAM16.

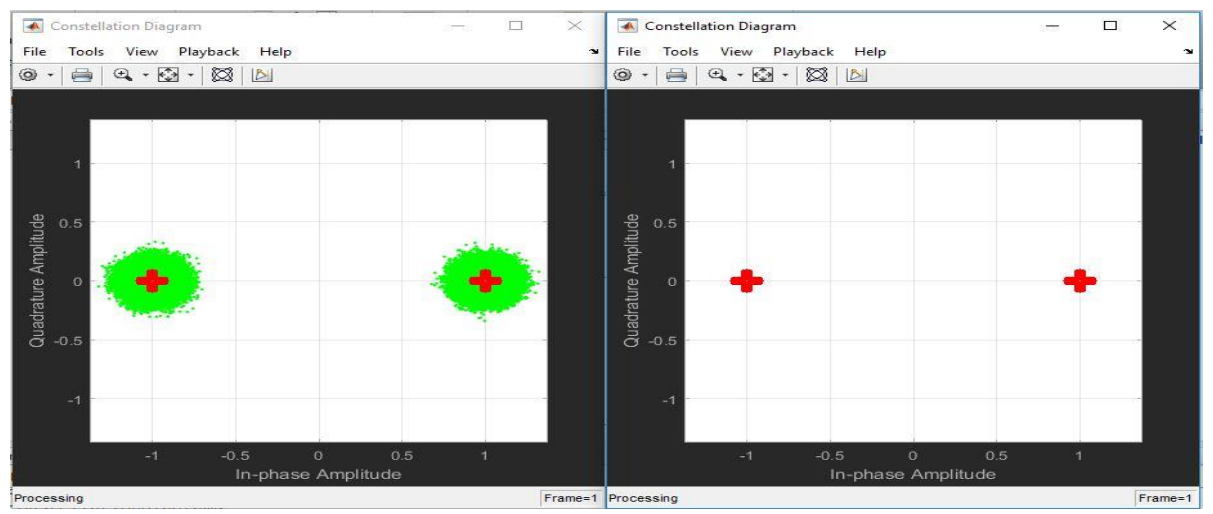

Fig. 10. PAM-2 constellation diagram without and with noise. 


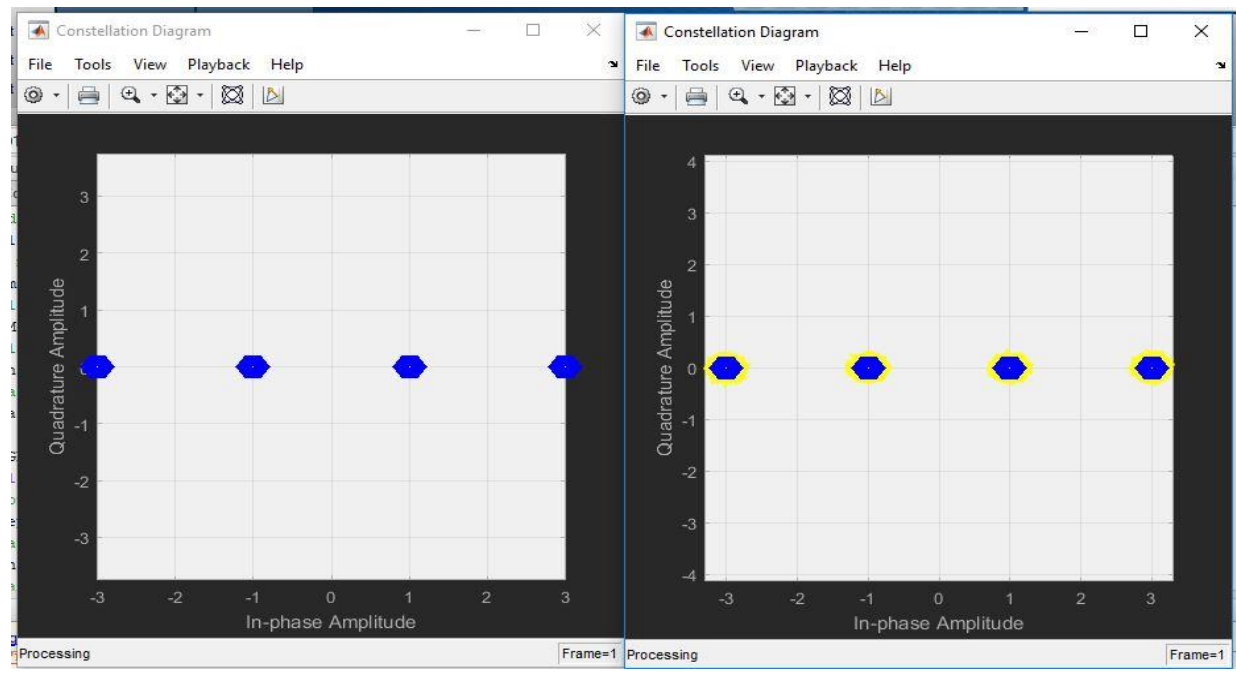

Fig. 11.PAM-4 constellation diagram without and with noise.

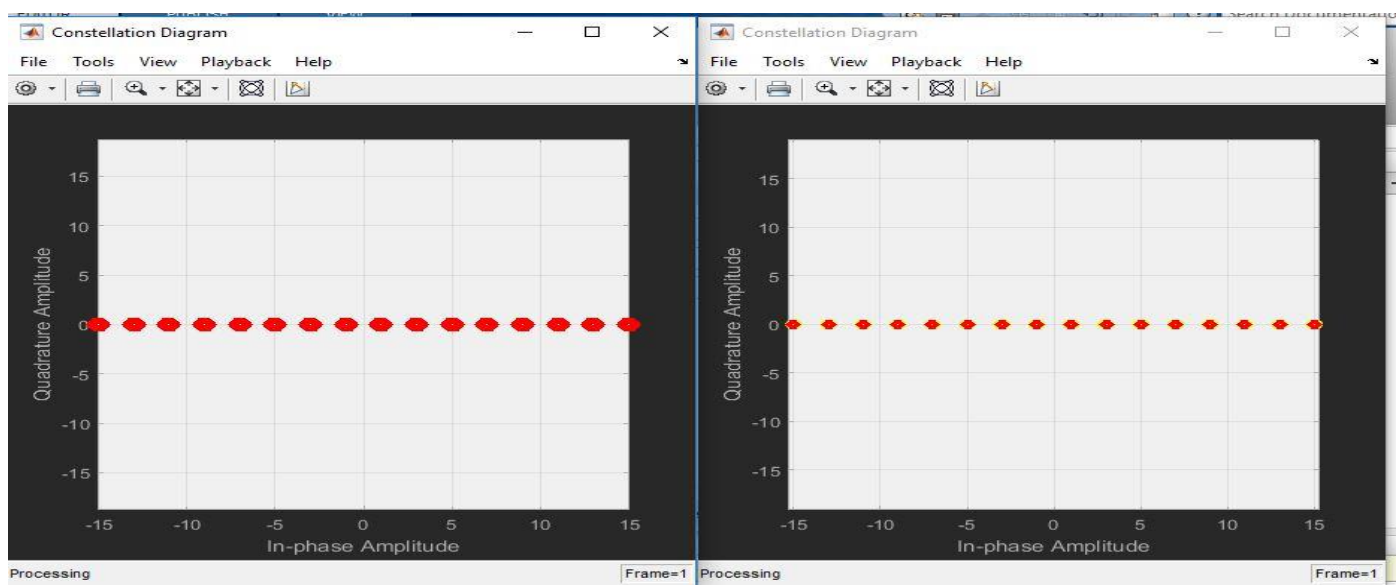

Fig. 12. PAM-16 constellation diagram without and with noise.

\subsection{Gray mapping of each signal}

Gray codes are coded binary representation of a decimal digit which changed in 1-bit position for consecutive digits. The following results obtained below Figures 13, 14 and 15 respectively shows the number of bits transmitted in various level of PAM as discussed.

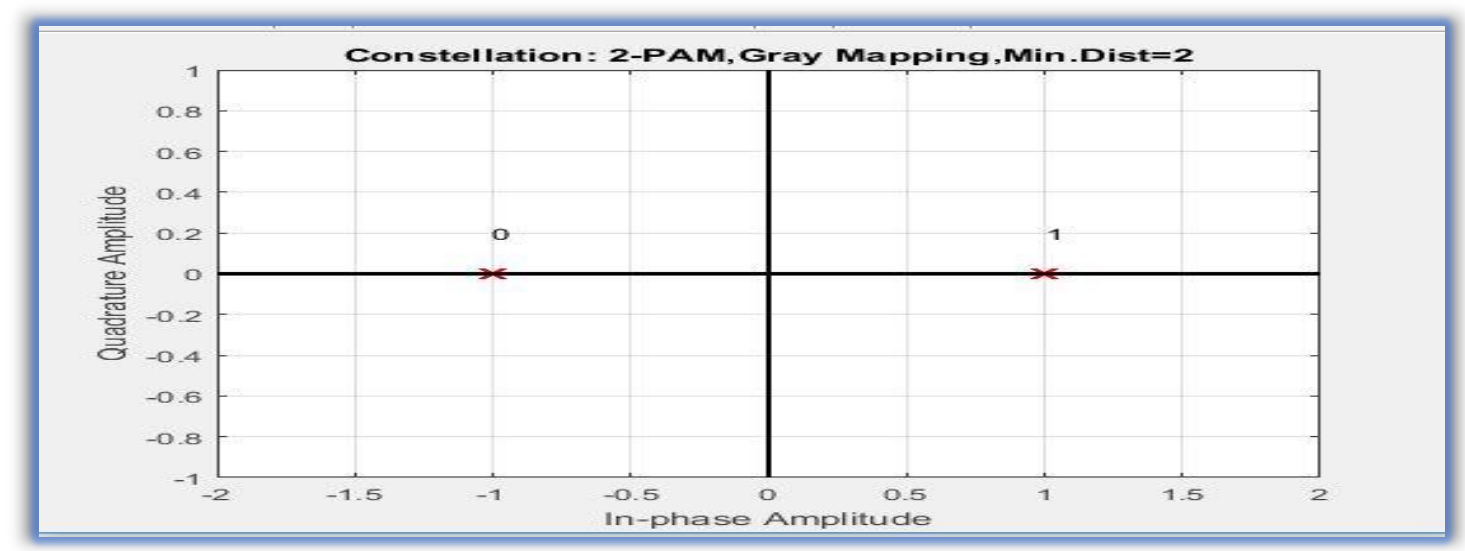

Fig. 13. Gray mapping for PAM-2. 


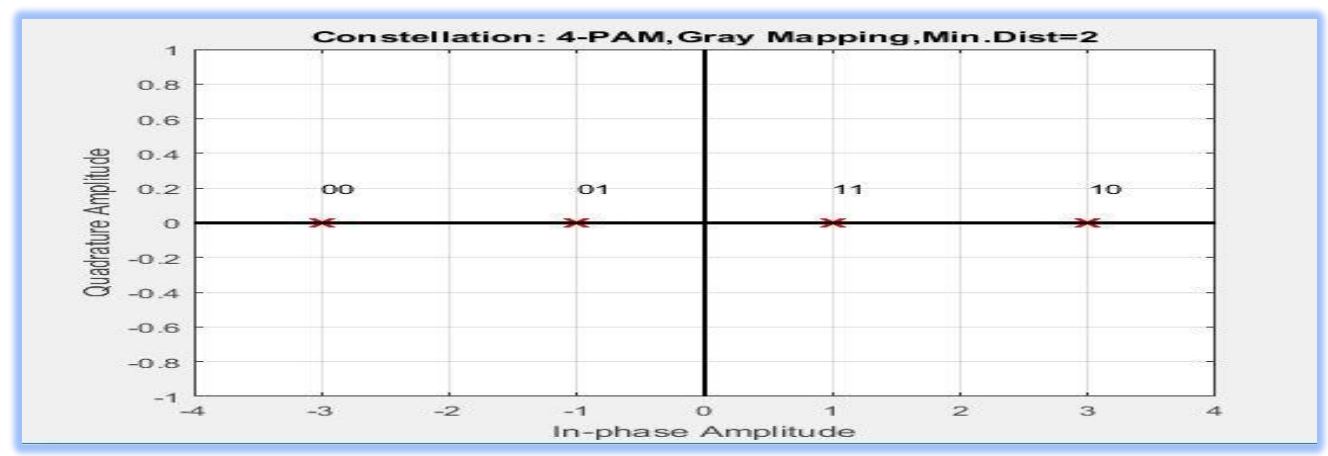

Fig. 14. Gray mapping for PAM-4.

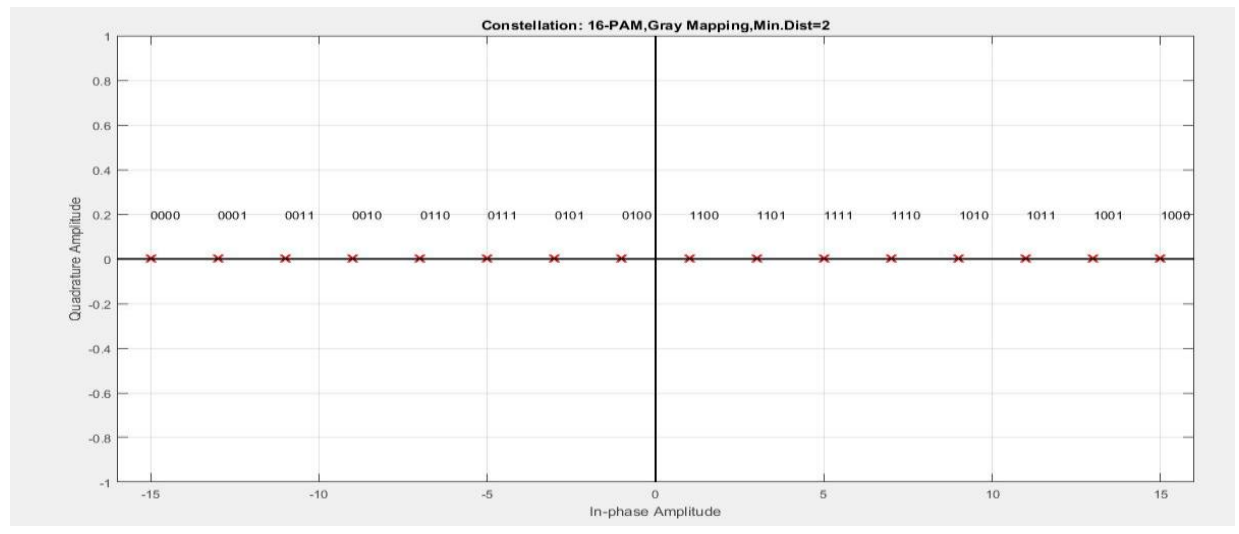

Fig. 15. Gray mapping for PAM-16.

\subsection{Bit Error Rate Comparison}

The study presented a joint bit error rate (BER) comparison of modulated PAM multilevel (M) of 2, 4 and 16 as revealed in Figure 16 below.

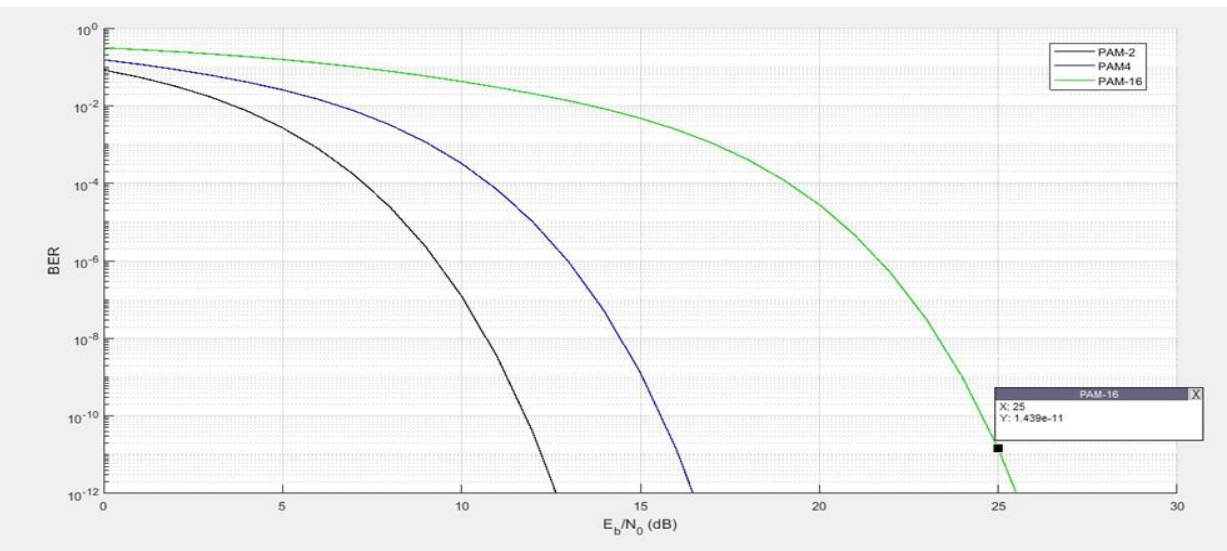

Fig. 16. Bit error rate curve of PAM-2, PAM-4 and PAM-16

The graph (Figure 16) shows the variation of the signal to noise ratio with the bit error rate (BER). Where signal to noise ratio indicates the strength of a signal in the presence of noise. The bit error rate indicates the ratio of the number of bits in error to the total bits transmitted.

From the three curves shown, it can be deduced that, for constant power, signal to noise ratio (SNR) increases as $M$ increases while; for constant BER, power increases as $M$ increases. The study revealed that as the $M$ increases, the faster the transmission and the smaller the bandwidth. In the consideration of the SNR of $25 \mathrm{db}$, the BER for PAM -16 is $1.435 e^{-11}$, this supported the statement above. 


\subsection{Hamming Code Detecting and Correcting Analysis}

The study improve the performance of the communication link by introduction of Hamming coding technique to reduce errors detected at random and channel noise as shown in Table 1, this was obtained from the MATLAB simulation which contains the transmitted bits without Hamming ( $\mathrm{TBnH})$, transmitted bits with hamming (TBwH), received bits without hamming $(\mathrm{TRBnH})$, received bits with hamming $(\mathrm{TRBwH})$, bit error rate without hamming $(\mathrm{BERnH})$, bit error rate with hamming $(\mathrm{BERwH})$.

Table 1. Errors detected from the random-generated signals without Hamming effect.

\begin{tabular}{|c|c|c|c|c|}
\hline & TBnH & TRBwH & EOH & BERnH \\
\hline message 1 & 6000 & 5869 & 131 & 0.0475 \\
\hline message2 & 6000 & 5868 & 132 & 0.050667 \\
\hline message 3 & 6000 & 5870 & 130 & 0.051667 \\
\hline message 4 & 6000 & 5851 & 149 & 0.056 \\
\hline message 5 & 6000 & 5877 & 123 & 0.048833 \\
\hline message 6 & 6000 & 5857 & 143 & 0.052 \\
\hline$=$ & & & & \\
\hline $6 \times 4$ table & & & & \\
\hline & TBnH & TRBnH & BIEnH & BERwH \\
\hline message 1 & 6000 & 5715 & 285 & 0.035 \\
\hline message2 & 6000 & 5696 & 304 & 0.039091 \\
\hline message 3 & 6000 & 5690 & 310 & 0.040909 \\
\hline message 4 & 6000 & 5664 & 336 & 0.0425 \\
\hline message 5 & 6000 & 5707 & 293 & 0.038636 \\
\hline message 6 & 6000 & 5688 & 312 & 0.038409 \\
\hline
\end{tabular}

Figure 17 and Figure 18 presented error in the generated signals with and without hamming code to compare the effect of noise.

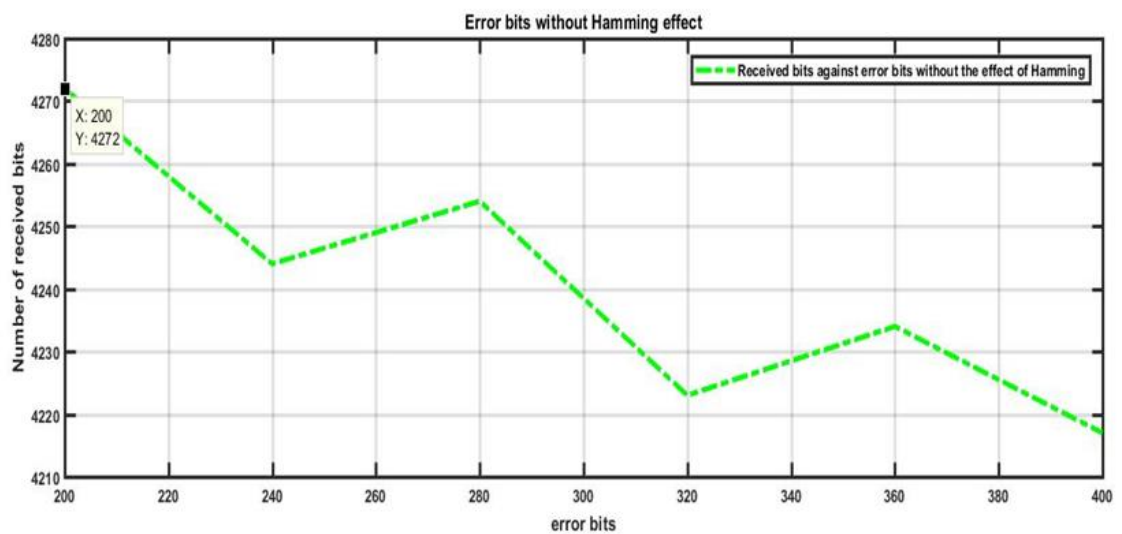

Fig. 17. Errors detected from the randomly generated signals without hamming effect. 


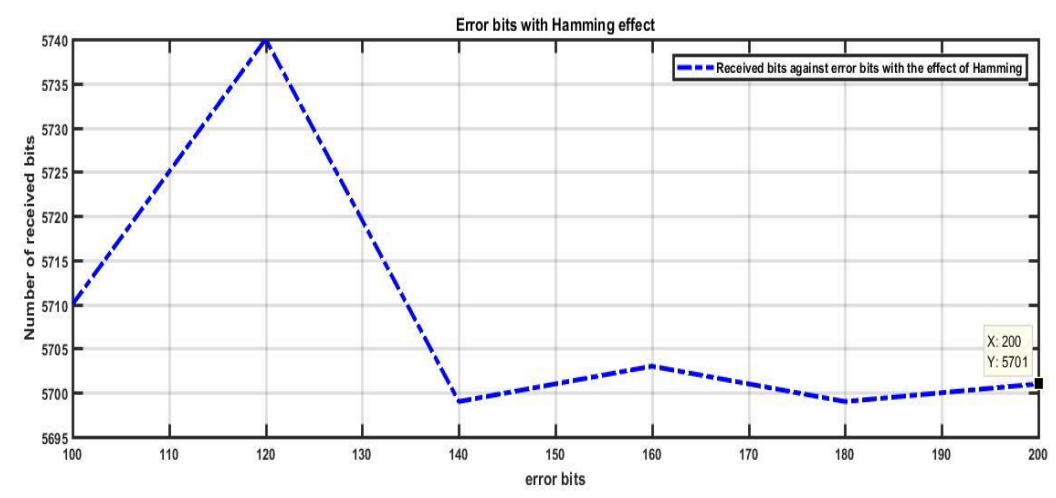

Fig. 18. Errors detected from the randomly generated signals with hamming effect.

From the results of the errors obtained from the generated signals, Figure 17 shows that for the same number of errors bits transmitted with the signal, the number of bits received without hamming coding is less than the number of bits received with hamming coding (Figure 18). This is evident if the cursor is at '200' (on the horizontal axis) is compared for the two graphs.

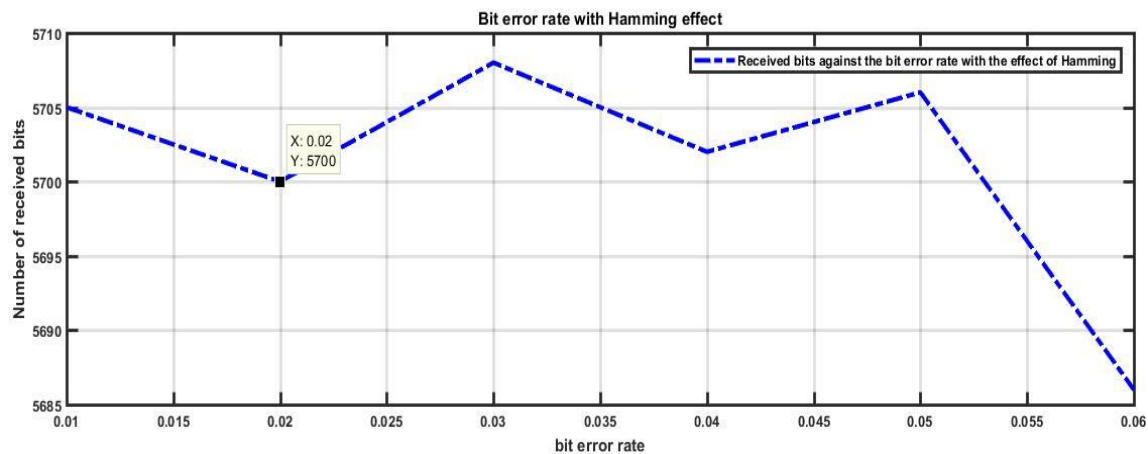

Fig. 19: Bit error rate with Hamming effect.

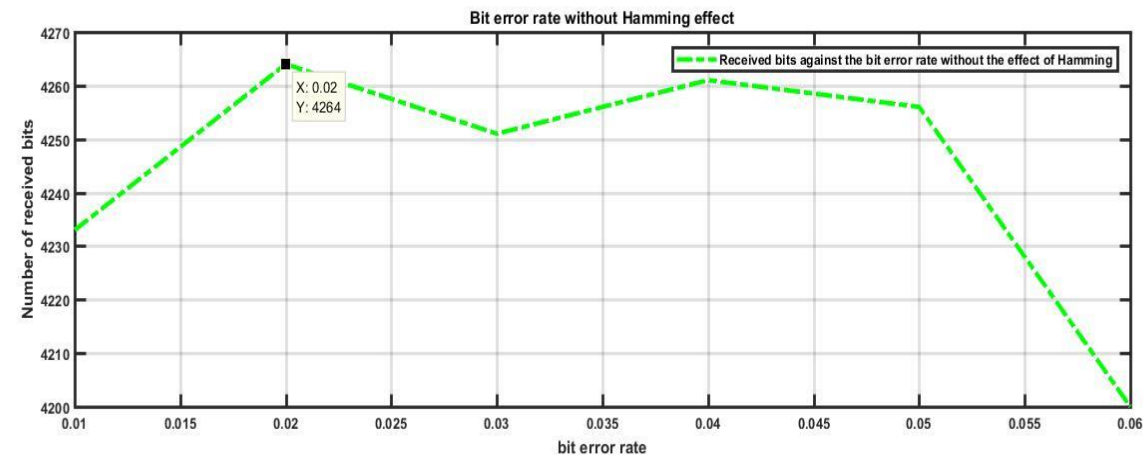

Fig. 20: Bit error rate without hamming effect.

Figure 19 and Figure 20 also show a plot of the received bits against the bit error rate with and without hamming code. The graphs reveal that for the same number of bit error rate, there is a significant difference in the number of the bits received. With hamming coding technique, more data bits are received at the receiver (without error) and if the hamming coding technique is not used the error received is a more and it increase and drops at some sharp point during transmission.

\section{CONCLUSION}

From the study of the research and simulations of the investigation, the following conclusions were deduced on comparison of PAM-16, PAM-4 and PAM-2 transmission:

a) PAM 16 has the lowest bandwidth. As a result of these, the rate of transmission of data is at the high side. 
b) PAM 2 and PAM 4 shows steeper bit error rate curves than that of PAM 16 modulated signal. This implies better accommodation for higher values of the signal to noise ratio and hence better signal fidelity at the receiving end.

c) With the use of Hamming coding technique in PAM 16, better bit error rate was achieved.

d) Reduced number of errors in transmission was obtained which provides better performance and efficiency of the system.

e) From the BER obtained, the integrity of the communication system can be determined.

f) The key parameter that is useful in accessing digital communication system can in the process of transmitting data from one point to another can also be obtained.

g) The investigation revealed a better signal quality can be achieved with improvement of coding system.

\section{REFERENCES}

[1] Čižmár, A., Networks in digital modulation schemes, 2018.

[2] Nielsen, A., 10G Ethernet IEEE 802.3an. Retrieved from http://www.ampnetconnect.eu, 2008.

[3] Apena, W.O., Performance evaluation of PAM-4 and PAM-2 modulation techniques using MATLAB, EJERS, 3(5), 2018, p. 36-40.

[4] Piranha, D., \& Led, R. G. B., Datasheet, 2007, p. 1-2. https://doi.org/10.1007/978-3-211-89836-9_355

[5] Shaw, S. Noise Terminology : Today's webinar important noise characteristics, 2018.

[6] Ungerboeck, G., 10GBASE - T : 10Gbit / s ethernet over copper, 2006, p. 20-22. 